Original Research Paper

\title{
Synthesis of Optimal Incoherent Subcarrier Tracking of BOC $(1,1)$ Navigation Signals
}

\author{
Alexandr Ivanovich Perov \\ National Research University, “Moscow Power Engineering Institute”, Moscow, Russia
}

Article history

Received: 22-06-2016

Revised: 11-09-2016

Accepted: 15-09-2016

Email: alexp@aha.ru

\begin{abstract}
Navigation receivers of global positioning systems that are operated in complex electromagnetic environment must have an increased noise immunity characteristic. One way of such noise immunity rising is to use incoherent operating mode of navigation receiver. There are known processing algorithms for navigation signals with Binary Phase Shift Keying (BPSK) modulation. But in the near-term outlook Global Navigation Satellite Systems (GLONASS), Global Positioning Systems (GPS) and Galileo known navigation signals with Binary Offset Carrier (BOC) modulation will be radiated. Processing algorithms for such signals are not described in scientific literature. So it is interesting to work out processing algorithms for BOC modulated signals in incoherent operating mode of navigation receiver. The article is devoted to synthesis and analysis of incoherent subcarrier tracking system under receiving BOC $(1,1)$ navigation signals. To synthesize an optimal tracking system, optimal estimation theory and dual estimation method are used that allow taking into account multi-peak a posteriori distribution of delay that caused by the periodic process (digital sinusoid) in the radio signal modulation function. When analyzing the synthesized estimation algorithm, the procedure for describing delay discriminators and dual variable discriminators were used in the form of a statistical equivalent, including discrimination and fluctuation characteristics. New equations have been obtained that describe optimal tracking system for incoherent mode of a navigation receiver. New expressions for delay and dual variable discriminators are derived. The article gives a scheme for these discriminators and formulas for their statistical characteristics. Simulated results are provided that illustrate accuracy characteristics enhancement. The obtained tracking algorithms may be used in the navigation consumer equipment of GLONASS, GPS, Galileo and other radio-navigation satellite systems operating with BOC(1.1) modulated signals.
\end{abstract}

Keywords: Radio-Navigation Satellite Systems, Incoherent Mode, Optimal Filtering, Navigation Receiver, BOC Modulation

\section{Introduction}

In modern satellite navigation systems such as GLONASS (Radiotekhnika, 2012; Perov and Kharisov, 2010), GPS (IS-GPS-200F, 2011; IS-GPS-800B, 2011), Galileo (European GNSS, 2010), BeiDou (BeiDou NSS, 2013 ) it is planned to use signals with binary offset carrier BOC $(1,1)$. Special feature of these signals is additional modulation by digital harmonic function. This function is periodical that leads to multi-peak a posteriori distribution of delay. When receiving navigation signal with BPSK modulation there is usually used Gaussian approximation of a posteriori distribution. In the case of receiving navigation signals with $\mathrm{BOC}$ modulation this approach is not totally rightful. It is desired to use methods that deal more correctly with multi-peak a posteriori distribution. So the task of synthesizing a delay estimation algorithm that takes into account multipeak a posteriori distribution is actual.

The coherent operational mode of a navigation receiver is described in a number of articles (BeiDou NSS, 2013; Martin et al., 2003; Heiries et al., 2004; Chen et al., 2006; Fine and Wilson, 1999; Fante, 2003; De Castro et al., 2006; Musso et al., 2006; Jovancevic et al., 
2007; Gyu-In et al., 2007; Jovanovic et al., 2012; Tikhionov and Kharisov, 1984; 2004; Hodgart et al., 2007 ; 2008). Some of them are devoted to signal acquisition problems. Spectral density of BOC modulated signals has two frequency lobes. So it is possible to process each of them separately using signal description as BPSK modulated. Such approach is used by Martin et al. (2003). Similar approach is exploited in (Heiries et al., 2004) for decision of the task of $\operatorname{BOC}(10,5)$ and $\operatorname{BOC}(14,2)$ acquisition. Chen et al. (2006), Fine and Wilson (1999) described tracking algorithms that are submitted more qualitatively but not from strict synthesis. In some papers the idea was utilized to use representation of signal derivative in delay as multi-strobe discriminator (Fante, 2003; De Castro et al., 2006). Using this approach one can manage with discriminatory characteristic but it cannot decide the problem of delay estimation ambiguity.

Multi-strobe discriminators are also treated by (Musso et al., 2006; Jovancevic et al., 2007; Gyu-In et al., 2007; Jovanovic et al., 2012). The idea to use dual delay estimations that are entered into the composition of code delay and phase was firstly described by Tikhionov and Kharisov (1984; 2004) for a synchronization problem. For satellite navigation tasks this approach is described in (Hodgart et al., 2007; 2008) where delay tracking of BOC modulated signals is treated. In these articles two delay estimations are formed and they are combined in overall estimation using this or that rule. Estimations of code delay and dual code delay tied with the digital subcarrier can be formed in the common tracking system (Tikhionov and Kharisov, 2004; Vinogradov et al., 2009). This structure of the tracking system follows from optimal estimation theory. All described articles result in algorithms with processing at carrier frequency as for delay so for phase tracking.

In the research carried out by Perov (2015) the basic idea of dual delay estimation is applied to synthesize optimal receiving of $\operatorname{BOC}(1,1)$ modulated signal in the coherent operating mode and processing at subcarrier frequencies. At the same time it is known that the coherent operating mode of navigation receiver is characterized by low noise immunity. Incoherent operating mode with known BPSK modulated signal delay tracking algorithms and processing at carrier frequencies is used to increase noise immunity of navigation consumer equipment (Perov and Kharisov, 2010). In this article incoherent operating mode of navigation receiver is investigated when a signal with BOC $(1,1)$ modulation is received and processing at subcarrier frequencies is desired.

In the article there are used following terminology (definitions).

Optimal tracking system-a tracking system synthesized using optimal filtering theory (Tikhionov and Kharisov, 2004; Perov, 2012).
Processing at subcarrier frequencies-processing in correlotors using reference signals at subcarrier frequencies.

Incoherent operating mode of navigation receiver-the mode of navigation receiver operation using a Delay Tracking Loop (DLL) and Frequency Tracking Loop (FLL).

\section{Setting the Synthesis Problem}

Let us consider navigation signal reception in discreet time $t_{k, i}$ (Fig. 1), where $t_{k, i}=k T+i T_{d} ; T=N T_{d}$-discrete processing step in tracking system loop; $T_{d}$-sampling period in ADC.

There is a following realization at the receiver input:

$y_{k, i}=s\left(t_{k, i}, \tau_{k}, \varphi_{k, i}\right)+n_{k, i}$

where, $\varphi_{k, i}$ is a signal phase; $n_{k, i}$-discrete white Gaussian noise with a dispersion of $\sigma_{n}^{2}=\frac{N_{0}}{2 T_{d}}$, where

$N_{0}$-receiver internal noise of one-way power spectral density.

It is assumed that the signal $s\left(t_{k, i}, \tau_{k}, \varphi_{k, i}\right)$ is $\operatorname{BOC}(1,1)$ modulated so its description is following:

$$
\begin{aligned}
& s\left(t_{k, i}, \tau_{k}\right)=A h_{c}\left(t_{k, i}-\tau_{k}\right) \\
& h_{d s}\left(t_{k, i}-\tau_{k}\right) h_{I D}\left(t_{k, i}-\tau_{k}\right) \cos \left(\omega_{0} t_{k, i}\right)
\end{aligned}
$$

where, $A$ is a signal amplitude; $\tau_{k}$-signal delay; $h_{c}\left(t_{k, i}\right)$ code range modulation function; $h_{d s}\left(t_{k, i}\right)=$ $\operatorname{sign}\left(\sin \left(2 \pi f_{s}\right)\right)$-subcarrier modulation function, where $f_{s}$ subcarrier frequency; $h_{D I}\left(t_{k, i}\right)$-navigation message.

When synthesizing the optimal algorithm of delay filtering it is supposed that for each time interval $\left[t_{k-1}, t_{k}\right]$ phase $\varphi_{k, i}$ is described by the expression:

$$
\varphi_{k, i}=\varphi_{k, 0}+i T_{d} \omega_{D, k}
$$

where, $\omega_{D, k}$ is Doppler frequency shift changing only at the clock time $t_{k}$.

It is supposed also that Doppler signal frequency shift is known and can be estimated in the receiver autonomously, i.e., it is assumed a known function. Initial phases $\varphi_{k, 0}$ for each time interval are unknown and assumed to be random values with equal distribution regularity at the interval $[-\pi, \pi]$.

The model of signal delay for the accepted assumptions may be written in the form:

$\tau_{k}=\tau_{k-1}-\omega_{D, k} T /\left(2 \pi f_{0}\right)+\xi_{\tau, k-1}$

where, $f_{0}$ is a signal carrier frequency; $\xi_{\tau, k-1}$-discrete white Gaussian noise with a dispersion of $D_{\xi}$ 


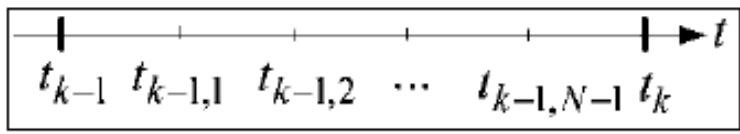

Fig. 1. Indexing time scheme

Let us formulate the task: It is necessary to synthesize optimal delay tracking system at the clock times $t_{k}$ processing observations (1) and taking into account delay model (3).

\section{The Synthesis of Optimal Delay Tracking Algorithm}

First of all it should be mentioned that since the rate of input counting (1) is higher than the $\tau_{k}$ rate of change it is required to use optimal filtering theory with grouping of observations (Perov and Kharisov, 2010).

Method of dual delay estimation is based on introducing of two variables one of which $\tau$ is connected with the range code and another variable $\tau_{d s}$ is connected with the subcarrier modulation function (Tikhionov and Kharisov, 2004; Perov, 2015). Using these variables it is possible to write down the signal (2) in the form:

$$
\begin{aligned}
& s\left(t_{k, i}, \tau_{k}, \tau_{d s, k}\right)=A h_{c}\left(t_{k, i}-\tau_{k}\right) h_{d s}\left(t_{k, i}-\tau_{d s, k}\right) \\
& h_{D I}\left(t_{k, i}-\tau_{k}\right) \cos \left(\omega_{0} t_{k, i}+\varphi_{k, 0}+i T_{d} \omega_{D, k}\right)
\end{aligned}
$$

Assume $\tau_{d s, k}=\tau_{k}$ for each $k$. Therefore, temporal variation of $\tau_{d s, k}$ is described with equation similar to (3).

Now the expanded vector $x=\left|\begin{array}{ll}\tau & \tau_{d s}\end{array}\right|^{T}$ should be introduced, for which the vector equation is written down as follows:

$$
x_{k}=F x_{k-1}+P u_{k}+G \xi_{\tau, k-1}
$$

Where:

$$
F=\left|\begin{array}{ll}
1 & 0 \\
0 & 1
\end{array}\right|, G=\left|\begin{array}{l}
1 \\
1
\end{array}\right|, P=\left|\begin{array}{l}
1 \\
1
\end{array}\right|, u_{k}=-\omega_{D, k} T /\left(2 \pi f_{0}\right)
$$

Following optimal filtering theory it is necessary to consider a posteriori density $p\left(x_{k} \mid Y_{0}^{k+1}\right)$, where $Y_{k, 1}^{k+1}=\left\{y_{k, 1}, y_{k, 2}, \ldots, y_{k, N}\right\}$ is observations received at time interval $\left[t_{k, 1}, t_{k+1}\right]$ (Fig. 1), for which the following equations can be written down (Perov, 2012):

$$
\begin{aligned}
& p\left(x_{k} \mid Y_{0}^{k+1}\right)=c p\left(x_{k} \mid Y_{0}^{k}\right) p\left(Y_{k, 1}^{k+1} \mid x_{k}\right), \\
& p\left(x_{k} \mid Y_{0}^{k}\right)=\int_{-\infty}^{\infty} p\left(x_{k-1} \mid Y_{0}^{k}\right) p\left(x_{k} \mid x_{k-1}\right) d x_{k-1}
\end{aligned}
$$

Note that observations $Y_{k, 1}^{k+1}$ depend on $\tau_{k}$ and $\tau_{d s, k}$ defined at the moment $t_{k}$. So it is necessary to consider a posteriori density of vector $x_{k}$ while processing observations $Y_{k, 1}^{k+1}$ and form an estimate of the state vector $\hat{x}_{k}$ that corresponds to the state vector $x_{k}$ at the time moment $t_{k}$.

A posteriori density $p\left(x_{k} \mid Y_{0}^{k+1}\right)$ is multi-peak. In (Perov and Kharisov, 2010; Tikhionov and Kharisov, 2004) two stage procedure is suggested. At the first stage it is necessary to consider the main lobe of a posteriori density and to use usual Gaussian approximation of this function. At the second stage the multi-peak form of a posteriori density is taken into account and correction of delay estimation is fulfilled.

Following this methodology let us write down equation for optimal filtering of $\hat{x}_{k}=E\left[x_{k}\right]$ vector (Perov and Kharisov, 2010; Hodgart et al., 2008; Perov, 2012).

$\hat{x}_{k}=\tilde{x}_{k}+D_{x, k}\left(\frac{\partial F_{k}\left(\tilde{x}_{k}\right)}{\partial x}\right)^{T}, \tilde{x}_{k}=F \hat{x}_{k-1}+P u_{k}$

$\tilde{D}_{x, k}=F D_{x, k-1} F^{T}+G D_{\xi} G^{T}, D_{x, k}^{-1}=\tilde{D}_{x, k}^{-1}-\frac{\partial}{\partial x}\left(\frac{\partial F_{k}\left(\tilde{x}_{k}\right)}{\partial x}\right)^{T}$

Where:

$\tilde{x}_{k}=$ State vector estimation; filtered process

$\tilde{x}_{k}=$ Extrapolated state vector estimation

$D_{x, k}=$ Dispersion matrix of filtration errors

$\tilde{D}_{x, k}=$ Dispersion matrix of extrapolation errors

$F_{k}\left(\tilde{x}_{k}\right)=\operatorname{Ln}\left(\frac{1}{2 \pi} \int_{-\pi}^{\pi} \exp \left(\tilde{F}_{k}\left(\tilde{x}_{k}\right)\right) d \varphi_{k, 0}\right)$

$\tilde{F}_{k}\left(x_{k}\right)=\frac{1}{\sigma_{n}^{2}} \sum_{i=1}^{N} y_{k, i} s\left(t_{k, i}, x_{k}\right)$

Here averaging on non-informative phase $\varphi_{k, 0}$ is performed.

Let us write down the right part of the formula (9) in the form:

$\tilde{F}_{k}\left(x_{k}\right)=\frac{1}{\sigma_{n}^{2}} \sum_{i=1}^{N_{p}} y_{k, l} s\left(t_{k, l}, x_{k}\right)=\frac{A}{\sigma_{n}^{2}} \sum_{l=1}^{N} y_{k, l} h_{c}\left(t_{k, l}-\tau_{k}\right) h_{d s}$
$\left(t_{k, l}-\tau_{d s, k}\right) h_{D I}\left(t_{k, i}-\tau_{k}\right) \cos \left(\omega_{0} t_{k, l}+\varphi_{k, 0}+(l-1) T_{d} \omega_{D, k}\right)$ 
For realization of subcarrier processing let us interchange $\operatorname{sign}\left(\sin \left(\Omega t_{k, l}\right)\right)$ with $\sin \left(\Omega t_{k, l}\right)$. While substituting it into reference signal $s\left(t_{k, l}, x_{k}\right)$ instead of $h_{d s}\left(t_{k, l}-\tau_{d s, k}\right)$ the following equation is obtained:

$$
\begin{aligned}
& s\left(t_{k, l}, x_{k}\right)=A h_{c}\left(t_{k, l}-\tau_{k}\right) h_{D I}\left(t_{k, l}-\tau_{k}\right) \\
& \sin \left(\Omega\left(t_{k, l}-\tau_{d s, k}\right)\right) \cos \left(\omega_{0} t_{k, l}+\omega_{D, k}(l-1) T_{d}+\varphi_{k, 0}\right)
\end{aligned}
$$

Let us introduce subcarrier frequencies:

$$
\begin{aligned}
& \omega_{s c 1}=\omega_{0}+\Omega \\
& \omega_{s c 2}=\omega_{0}-\Omega
\end{aligned}
$$

and present the product of trigonometric functions in (11) as:

$$
\begin{aligned}
& \sin \left(\Omega\left(t_{k, l}-\tau_{d s, k}\right)\right) \cos \left(\omega_{0} t_{k, l}+\omega_{D, k}(l-1) T_{d}+\varphi_{k, 0}\right) \\
& =\frac{1}{2}\left[\begin{array}{l}
\sin \left(\omega_{s c 1} t_{k, l}+\omega_{D, k}(l-1) T_{d}+\varphi_{k, 0}-\Omega \tau_{d s, k}\right) \\
-\sin \left(\omega_{s c 2} t_{k, l}+\omega_{D, k}(l-1) T_{d}+\varphi_{k, 0}+\Omega \tau_{d s, k}\right)
\end{array}\right]
\end{aligned}
$$

While introducing (13) into (10) the following expression is obtained:

$$
\begin{aligned}
& \tilde{F}_{k}\left(x_{k}\right)=\frac{1}{\sigma_{n}^{2}} \sum_{l=1}^{N_{p}} y_{k, l} s\left(t_{k, l}, x_{k}\right) \\
& -\frac{A}{2 \sigma^{2}}\left[\begin{array}{l}
\sum_{l=1}^{N} y_{k, l} h_{c}\left(t_{k, l}-\tau_{k}\right) h_{D I}\left(t_{k, i}-\tau_{k}\right) \\
\sin \left(\omega_{s c l} t_{k, l}+\omega_{D, k}(l-1) T_{d}+\varphi_{k, 0}-\Omega \tau_{d s, k}\right) \\
-\sum_{l=1}^{N} y_{k, l} h_{c}\left(t_{k, l}-\tau_{k}\right) h_{D I}\left(t_{k, l}-\tau_{k}\right) \\
\sin \left(\omega_{s c 2} t_{k, l}+\omega_{D, k}(l-1) T_{d}+\varphi_{k, 0}+\Omega \tau_{d s, k}\right)
\end{array}\right]
\end{aligned}
$$

There are two summands in (14):

$$
\begin{aligned}
& \tilde{F}_{1, k}\left(x_{k}\right)=\frac{A}{2 \sigma_{n}^{2}} \sum_{l=1}^{N} y_{k, l} h_{c}\left(t_{k, l}, x_{k}\right) h_{D I}\left(t_{k, l}-\tau_{k}\right) \\
& \sin \left(\omega_{s c 1} t_{k, l}+\omega_{D, k}(l-1) T_{d}+\varphi_{k, 0}-\Omega \tau_{d s, k}\right) \\
& \tilde{F}_{2, k}\left(x_{k}\right)=\frac{A}{2 \sigma_{n}^{2}} \sum_{l=1}^{N} y_{k, l} h_{c}\left(t_{k, l}-\tau_{k}\right) h_{D I}\left(t_{k, l}-\tau_{k}\right) \\
& \sin \left(\omega_{s c 2} t_{k, l}+\omega_{D, k}(l-1) T_{d}+\varphi_{k, 0}+\Omega \tau_{d s, k}\right)
\end{aligned}
$$

Along with this

$$
\tilde{F}_{k}\left(x_{k}\right)=\tilde{F}_{1, k}\left(x_{k}\right)+\tilde{F}_{2, k}\left(x_{k}\right)
$$

Let us consider $\tilde{F}_{1, k}\left(x_{k}\right)$ and transform the trigonometric function:

$$
\begin{aligned}
& \sin \left(\omega_{s c l} t_{k, l}+\omega_{D, k}(l-1) T_{d}+\varphi_{k, 0}-\Omega \tau_{d s, k}\right) \\
& =\sin \left(\omega_{s c l} t_{k, l}+\omega_{D, k}(l-1) T_{d}\right) \cos \left(\varphi_{k, 0}-\Omega \tau_{d s, k}\right) \\
& +\cos \left(\omega_{s c l} t_{k, l}+\omega_{D, k}(l-1) T_{d}\right) \sin \left(\varphi_{k, 0}-\Omega \tau_{d s, k}\right)
\end{aligned}
$$

Designating:

$$
\psi_{s c 1}\left(\tau_{d s, k}\right)=\varphi_{k, 0}-\Omega \tau_{d s, k}
$$

It is possible to introduce (16) into the formula for $\tilde{F}_{1, k}\left(x_{k}\right)$ and write down:

$$
\begin{aligned}
& \tilde{F}_{1, k}\left(x_{k}\right) \\
& =\frac{A}{2 \sigma_{n}^{2}}\left[\begin{array}{l}
\cos \left(\psi_{s c l}\left(\tau_{d s, k}\right)\right) \sum_{l=1}^{N} y_{k, l} h_{c}\left(t_{k, l}-\tau_{k}\right) \\
h_{D I}\left(t_{k, i}-\tau_{k}\right) \sin \left(\omega_{s c l} t_{k, l}+\omega_{D, k}(l-1) T_{d}\right) \\
+\sin \left(\psi_{s c l}\left(\tau_{d s, k}\right)\right) \sum_{l=1}^{N} y_{k, l} h_{c}\left(t_{k, l}-\tau_{k}\right) \\
h_{D I}\left(t_{k, i}-\tau_{k}\right) \cos \left(\omega_{s c l} t_{k, l}+\omega_{D, k}(l-1) T_{d}\right)
\end{array}\right]
\end{aligned}
$$

Let us introduce correlation sums (coherent and squared ones) at the subcarrier frequency $\omega_{s c 1}$ :

$$
\begin{aligned}
& I_{s c l, k}\left(\tau_{k}\right)=\frac{A}{2 \sigma_{n}^{2}} \sum_{l=1}^{N} y_{k, l} h_{c}\left(t_{k, l}-\tau_{k}\right) \\
& h_{D I}\left(t_{k, l}-\tau_{k}\right) \cos \left(\omega_{s c l} t_{k, l}+\omega_{D, k}(l-1) T_{d}\right), \\
& Q_{s c l, k}\left(\tau_{k}\right)=\frac{A}{2 \sigma_{n}^{2}} \sum_{l=1}^{N} y_{k, l} h_{c}\left(t_{k, l}-\tau_{k}\right) \\
& h_{D I}\left(t_{k, l}-\tau_{k}\right) \sin \left(\omega_{s c l} t_{k, l}+\omega_{D, k}(l-1) T_{d}\right)
\end{aligned}
$$

and write down (17) in the form:

$$
\begin{aligned}
& \tilde{F}_{1, k}\left(x_{k}\right)=\cos \left(\psi_{s c 1}\left(\tau_{d s, k}\right)\right) Q_{s c l, k}\left(\tau_{k}\right) \\
& +\sin \left(\psi_{s c 1}\left(\tau_{d s, k}\right)\right) I_{s c l, k}\left(\tau_{k}\right)
\end{aligned}
$$

Introduce:

$$
X_{s c l, k}\left(\tau_{k}\right)=\sqrt{I_{s c l, k}^{2}\left(\tau_{k}\right)+Q_{s c l, k}^{2}\left(\tau_{k}\right)}
$$

and present (19) as:

$$
\tilde{F}_{1, k}\left(x_{k}\right)=X_{s c 1, k}\left(\tau_{k}\right) \cos \left(\psi_{s c 1}\left(\tau_{d s, k}\right)-\hat{\psi}_{s c 1}\right)
$$

Where:

$$
\cos \left(\hat{\psi}_{s c 1}\right)=\frac{Q_{s c 1, k}\left(\tau_{k}\right)}{X_{s c l, k}\left(\tau_{k}\right)}, \sin \left(\hat{\psi}_{s c 1}\right)=\frac{I_{s c 1, k}\left(\tau_{k}\right)}{X_{s c l, k}\left(\tau_{k}\right)}
$$


Let us consider $\tilde{F}_{2, k}\left(x_{k}\right)$ similarly and transform the trigonometric function under the sign of sum:

$$
\begin{aligned}
& \sin \left(\omega_{s c 2} t_{k, l}+\omega_{D, k}(l-1) T_{d}+\varphi_{k, 0}+\Omega \tau_{d s, k}\right) \\
& =\sin \left(\omega_{s c 2} t_{k, l}+\omega_{D, k}(l-1) T_{d}\right) \cos \left(\varphi_{k, 0}+\Omega \tau_{d s, k}\right) \\
& +\cos \left(\omega_{s c 2} t_{k, l}+\omega_{D, k}(l-1) T_{d}\right) \sin \left(\varphi_{k, 0}+\Omega \tau_{d s, k}\right)
\end{aligned}
$$

Designating:

$$
\psi_{s c 2}\left(\tau_{d s, k}\right)=\varphi_{k, 0}+\Omega \tau_{d s, k}
$$

It is possible to introduce (23) into the formula for $\tilde{F}_{2, k}\left(x_{k}\right)$ and write down:

$$
\tilde{F}_{2, k}\left(x_{k}\right)=\frac{-A}{2 \sigma_{n}^{2}}\left[\begin{array}{l}
\cos \left(\psi_{s c 2}\left(\tau_{d s, k}\right)\right) \sum_{l=1}^{N} y_{k, l} h_{c}\left(t_{k, l}-\tau_{k}\right) h_{D I} \\
\left(t_{k, l}-\tau_{k}\right) \sin \left(\omega_{s c 2} t_{k, l}+\omega_{D, k}(l-1) T_{d}\right) \\
+\sin \left(\psi_{s c 2}\left(\tau_{d s, k}\right)\right) \sum_{l=1}^{N} y_{k, l} h_{c}\left(t_{k, l}-\tau_{k}\right) h_{D I} \\
\left(t_{k, l}-\tau_{k}\right) \cos \left(\omega_{s c 2} t_{k, l}+\omega_{D, k}(l-1) T_{d}\right)
\end{array}\right]
$$

Let us introduce correlation sums (coherent and squared ones) at the subcarrier frequency $\omega_{s c 2}$ :

$$
\begin{aligned}
& I_{s c 2, k}\left(\tau_{k}\right)=\frac{-A}{2 \sigma_{n}^{2}} \sum_{i=1}^{N} y_{k, l} h_{c}\left(t_{k, l}-\tau_{k}\right) \\
& h_{D I}\left(t_{k, l}-\tau_{k}\right) \cos \left(\omega_{s c 2} t_{k, l}+\omega_{D, k}(l-1) T_{d}\right), \\
& Q_{s c 2, k}\left(\tau_{k}\right)=\frac{-A}{2 \sigma_{n}^{2}} \sum_{i=1}^{N} y_{k, l} h_{c}\left(t_{k, l}-\tau_{k}\right) \\
& h_{D I}\left(t_{k, l}-\tau_{k}\right) \sin \left(\omega_{s c 2} t_{k, l}+\omega_{D, k}(l-1) T_{d}\right)
\end{aligned}
$$

and write down (25) in the form:

$$
\tilde{F}_{2, k}\left(x_{k}\right)=X_{s c 2, k}\left(\tau_{k}\right) \cos \left(\psi_{s c 2}\left(\tau_{d s, k}\right)-\hat{\psi}_{s c 2}\right)
$$

Where:

$$
\begin{aligned}
& X_{s c 2 k}\left(\tau_{k}\right)=\sqrt{I_{s c 2, k}^{2}\left(\tau_{k}\right)+Q_{s c 2, k}^{2}\left(\tau_{k}\right)} \\
& \cos \left(\hat{\psi}_{s c 2}\right)=\frac{Q_{s c 2, k}\left(\tau_{k}\right)}{X_{s c 2, k}\left(\tau_{k}\right)}, \sin \left(\hat{\psi}_{s c 2}\right)=\frac{I_{s c 2, k}\left(\tau_{k}\right)}{X_{s c 2, k}\left(\tau_{k}\right)}
\end{aligned}
$$

With regard to the obtained expressions (15) can be written down in the form:

$$
\begin{aligned}
& \tilde{F}_{k}\left(x_{k}\right)=X_{s c 1, k}\left(\tau_{k}\right) \cos \left(\psi_{s c 1}\left(\tau_{d s 1}\right)-\hat{\psi}_{s c 1}\right) \\
& -X_{s c 2, k}\left(\tau_{k}\right) \cos \left(\psi_{s c 2}\left(\tau_{d s, k}\right)-\hat{\psi}_{s c 2}\right)
\end{aligned}
$$

Now in (30) dependence on $\varphi_{k, 0}$ is extracted explicitly:

$$
\begin{aligned}
& \tilde{F}_{k}\left(x_{k}\right)=\cos \left(\varphi_{k, 0}\right)\left[\begin{array}{l}
X_{s c l, k}\left(\tau_{k}\right) \cos \left(\Omega \tau_{d s, k}+\hat{\psi}_{s c 1}\right) \\
+X_{s c 2, k}\left(\tau_{k}\right) \cos \left(\Omega \tau_{d s, k}-\hat{\psi}_{s c 2}\right)
\end{array}\right] \\
& +\sin \left(\varphi_{k, 0}\right)\left[\begin{array}{l}
X_{s c l, k}\left(\tau_{k}\right) \sin \left(\Omega \tau_{d s, k}+\hat{\psi}_{s c 1}\right) \\
+X_{s c 2, k}\left(\tau_{k}\right) \sin \left(\Omega \tau_{d s, k}-\hat{\psi}_{s c 2}\right)
\end{array}\right]
\end{aligned}
$$

Let us introduce a variable:

$$
\begin{aligned}
& Z_{k}^{2}=\left(\begin{array}{l}
X_{s c l, k}\left(\tau_{k}\right) \cos \left(\Omega \tau_{d s, k}+\hat{\psi}_{s c 1}\right) \\
+X_{s c 2, k}\left(\tau_{k}\right) \cos \left(\Omega \tau_{d s, k}-\hat{\psi}_{s c 2}\right)
\end{array}\right)^{2} \\
& +\left(\begin{array}{l}
X_{s c l, k}\left(\tau_{k}\right) \sin \left(\Omega \tau_{d s, k}+\hat{\psi}_{s c 1}\right) \\
+X_{s c 2, k}\left(\tau_{k}\right) \sin \left(\Omega \tau_{d s, k}-\hat{\psi}_{s c 2}\right)
\end{array}\right)
\end{aligned}
$$

and estimate $\hat{\varphi}_{0}$ with formulas:

$$
\begin{aligned}
& \cos \left(\hat{\varphi}_{0}\right)=\frac{1}{Z_{k}}\left(\begin{array}{l}
X_{s c 1, k}\left(\tau_{k}\right) \cos \left(\Omega \tau_{d s, k}+\hat{\psi}_{s c 1}\right) \\
+X_{s c 2, k}\left(\tau_{k}\right) \cos \left(\Omega \tau_{d s, k}-\hat{\psi}_{s c 2}\right)
\end{array}\right), \\
& \sin \left(\hat{\varphi}_{0}\right)=\frac{1}{Z_{k}}\left(\begin{array}{l}
X_{s c 1, k}\left(\tau_{k}\right) \sin \left(\Omega \tau_{d s, k}+\hat{\psi}_{s c 1}\right) \\
-X_{s c 2, k}\left(\tau_{k}\right) \sin \left(\Omega \tau_{d s, k}-\hat{\psi}_{s c 2}\right)
\end{array}\right)
\end{aligned}
$$

Which corresponds to:

$$
\hat{\varphi}_{0}=\operatorname{arctg}\left(\begin{array}{l}
X_{s c l, k}\left(\tau_{k}\right) \sin \left(\Omega \tau_{d s, k}+\hat{\psi}_{s c 1}\left(\tau_{k}\right)\right) \\
-X_{s c 2, k}\left(\tau_{k}\right) \sin \left(\Omega \tau_{d s, k}-\hat{\psi}_{s c 2}\left(\tau_{k}\right)\right) \\
X_{s c l, k}\left(\tau_{k}\right) \cos \left(\Omega \tau_{d s, k}+\hat{\psi}_{s c 1}\left(\tau_{k}\right)\right) \\
+X_{s c 2, k}\left(\tau_{k}\right) \sin \left(\Omega \tau_{d s, k}-\hat{\psi}_{s c 2}\left(\tau_{k}\right)\right)
\end{array}\right)
$$

Then (31) can be written in the form:

$$
\tilde{F}_{k}\left(x_{k}\right)=Z_{k}\left[\begin{array}{l}
\cos \left(\varphi_{k, 0}\right) \cos \left(\hat{\varphi}_{0}\right)+\sin \left(\varphi_{k, 0}\right) \\
\sin \left(\hat{\varphi}_{0}\right)=Z_{k} \cos \left(\varphi_{k, 0}-\hat{\varphi}_{0}\right)
\end{array}\right]
$$

Substitute (35) into (8) and perform averaging over $\varphi_{k-1,0}$ and unknown $h_{D I}$ values:

$$
\begin{aligned}
& F_{k}\left(x_{k}\right)=\operatorname{Ln}\left(\frac{1}{2 \pi} \int_{-\pi}^{\pi} \exp \left(Z_{k} \cos \left(\varphi_{k, 0}-\hat{\varphi}_{0}\right)\right) d \varphi_{k, 0}\right) \\
& =\operatorname{Ln}\left(I_{0}\left(Z_{k}\left(\tau_{k}, \tau_{d s, k}\right)\right)\right)
\end{aligned}
$$

Transpose (32) to the form of: 


$$
\begin{aligned}
& Z_{k}^{2}=X_{s c 1, k}^{2}\left(\tau_{k}\right)+X_{s c 2, k}^{2}\left(\tau_{k}\right)+2 X_{s c 1, k}\left(\tau_{k}\right) \\
& X_{s c 2, k}\left(\tau_{k}\right) \cos \left(\hat{\psi}_{s c 2}-\hat{\psi}_{s c 1}-2 \Omega \tau_{d s, k}\right)
\end{aligned}
$$

Averaged likelihood function (36) is formed using correlation sums (18), (26) with reference signals at subcarrier frequencies. In these correlation sums in the reference harmonic signals by the end of the accumulation interval (time moment $t_{k}$ ) phase $\varphi_{D, k}=\omega_{D, k-1} T$ is formed. Physically this phase remains in the reference signal and 'moves' to the next interval of the correlator operation. In the accepted synthesis procedure at the next internal of the correlator operation the phase $\tau_{D, k}$ is included into the initial phase under which averaging is performed, eliminating thereby the reference signal dependence on $\varphi_{D, k}$. In the section dealing with the analysis it will be shown that additional phase $\varphi_{D, k}$ available in the reference signals of the correlators does not affect the statistical characteristics of $Z_{k}$ and, consequently, the characteristics of the delay discriminator synthesized later on.

In the Equation 6 there is the derivative $\frac{\partial F_{k}\left(\tilde{x}_{k}\right)}{\partial x}$ that can be expressed in terms of discriminatory functions. According to the definition (Perov and Kharisov, 2010), discriminatory function on parameter $\lambda$ is described as:

$$
u_{d \lambda, k}=\left.\frac{\partial \tilde{F}_{k}\left(\tilde{x}_{k}\right)}{\partial \lambda}\right|_{x_{k}=\tilde{x}_{k}}
$$

In the task under consideration filtering system is

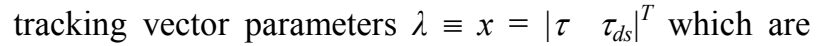
explicitly included into the signal function $s\left(t_{k, l}, x_{k}\right)$. Therefore, there are two discriminated parameters $\lambda=\mid \tau$ $\left.\tau_{d s}\right|^{T}$ and $\mathrm{u}_{d \lambda, k}$ is a vector discriminator.

Parameter $\tau$ is a range code delay. So the discriminator on this parameter will be named a code delay discriminator. For this discriminator it is possible to write:

$$
u_{d \tau, k}=\left.\frac{\partial}{\partial \tau}\left[\frac{1}{\sigma_{n}^{2}} \sum_{l=1}^{N} y_{k, l} s\left(t_{k, l}, x_{k}\right)\right]\right|_{x_{k}=\tilde{x}_{k}} \approx \frac{\partial Z_{k}\left(\tilde{\tau}_{k}\right)}{\partial \tau}
$$

Formula (38) can be simplified if we suppose $\cos \left(\hat{\psi}_{s c 2}-\hat{\psi}_{s c 1}-2 \Omega \tau_{d s, k}\right) \approx 1$ which holds true at a high tracking accuracy. Using this approximation formula (38) can be written in the form:

$$
u_{d \tau, k}=\frac{\partial X_{s c 1, k}\left(\tilde{\tau}_{k}\right)}{\partial \tau}+\frac{\partial X_{s c 2, k}\left(\tilde{\tau}_{k}\right)}{\partial \tau}
$$

In navigation the receiver usually introduces approximation of the derivative on delay:

$$
\begin{aligned}
& u_{d \tau, k}=\frac{\partial X_{s c 1(2), k}\left(\tilde{\tau}_{k}\right)}{\partial \tau} \\
& \approx \frac{X_{s c 1(2), k}\left(\tilde{\tau}_{k}+\Delta \tau / 2\right)-X_{s c 1(2), k}\left(\tilde{\tau}_{k}-\Delta \tau / 2\right)}{\Delta \tau}
\end{aligned}
$$

where, $\Delta \tau$ is the misalignment between reference signals, between range codes $h_{c}\left(t_{k, l}-\tau_{k}\right)$ of these reference signals.

A scheme that implements range code discriminator is shown in Fig. 2, where $I_{s c 1 E, k}\left(\tilde{\tau}_{k}\right), I_{s c 2 E, k}\left(\tilde{\tau}_{k}\right)$, $Q_{s c 1 E, k}\left(\tilde{\tau}_{k}\right), \quad Q_{s c 2 E, k}\left(\tilde{\tau}_{k}\right), \quad I_{s c 1 L, k}\left(\tilde{\tau}_{k}\right), \quad I_{s c 2 L, k}\left(\tilde{\tau}_{k}\right)$, $Q_{s c 1 L, k+1}\left(\tilde{\tau}_{k}\right), Q_{s c 2 L, k}\left(\tilde{\tau}_{k}\right)$-early and late components of correlators (18), (26).

Parameter $\tau_{d s}$ describes a delay that is entered into the subcarrier modulation function. So the discriminator on this parameter will be named a dual delay discriminator. For this discriminator it can be written:

$$
\begin{aligned}
& u_{d \tau_{d s}, k}=\left.\frac{\partial}{\partial \tau_{d s}}\left[\frac{1}{\sigma_{n}^{2}} \sum_{l=1}^{N_{p}} y_{k, l} s\left(t_{k, l}, x_{k}\right)\right]\right|_{x_{k}=\tilde{x}_{k}} \\
& =2 \Omega \frac{X_{s c 1, k}\left(\tilde{\tau}_{k}\right) X_{s c 2, k}\left(\tilde{\tau}_{k}\right)}{Z_{k}\left(\tilde{\tau}_{k}\right)} \sin \left(\hat{\psi}_{s c 2}-\psi_{s c 1}-2 \Omega \tilde{\tau}_{d s, k}\right)
\end{aligned}
$$

The structural scheme of the dual delay discriminator is shown in Fig. 3.

Function (40) includes harmonic function that is periodical on its argument, i.e., $\tau_{d s}$ parameter. Its stable points correspond to the condition $\hat{\psi}_{s c 1}-\psi_{s c 2}-2 \Omega \tilde{\tau}_{d s}=2 \pi m$ or:

$\hat{\tau}_{d s}=\frac{1}{\Omega}\left(\frac{\hat{\psi}_{s c 2}-\psi_{s c 1}}{2}+\pi m\right) \tilde{\tau}_{d s 0}+\frac{T_{\Omega} m}{2}$

where, $T_{\Omega}=\frac{2 \pi}{2}$ is a fluctuation period of subcarrier frequency: $\hat{\tau}_{d s 0}=\frac{\hat{\psi}_{s c 2}-\psi_{s c 1}}{2 \Omega}$.

Ambiguity of the estimation $\hat{\tau}_{d s}$ is conditioned by the parameter $m$ in (41). Method of dual variable gives the following expression for estimation of $m$ parameter (Tikhionov and Kharisov, 1984; 2004):

$$
\hat{m}=\arg \left(\min _{m}\left(\left|\hat{\tau}-\hat{\tau}_{d s}\right|\right)\right)=\arg \left(\min _{m}\left(\left|\hat{\tau}-\left(\hat{\tau}_{d s 0}(\hat{\tau})+\frac{T_{\Omega} m}{2}\right)\right|\right)\right)
$$




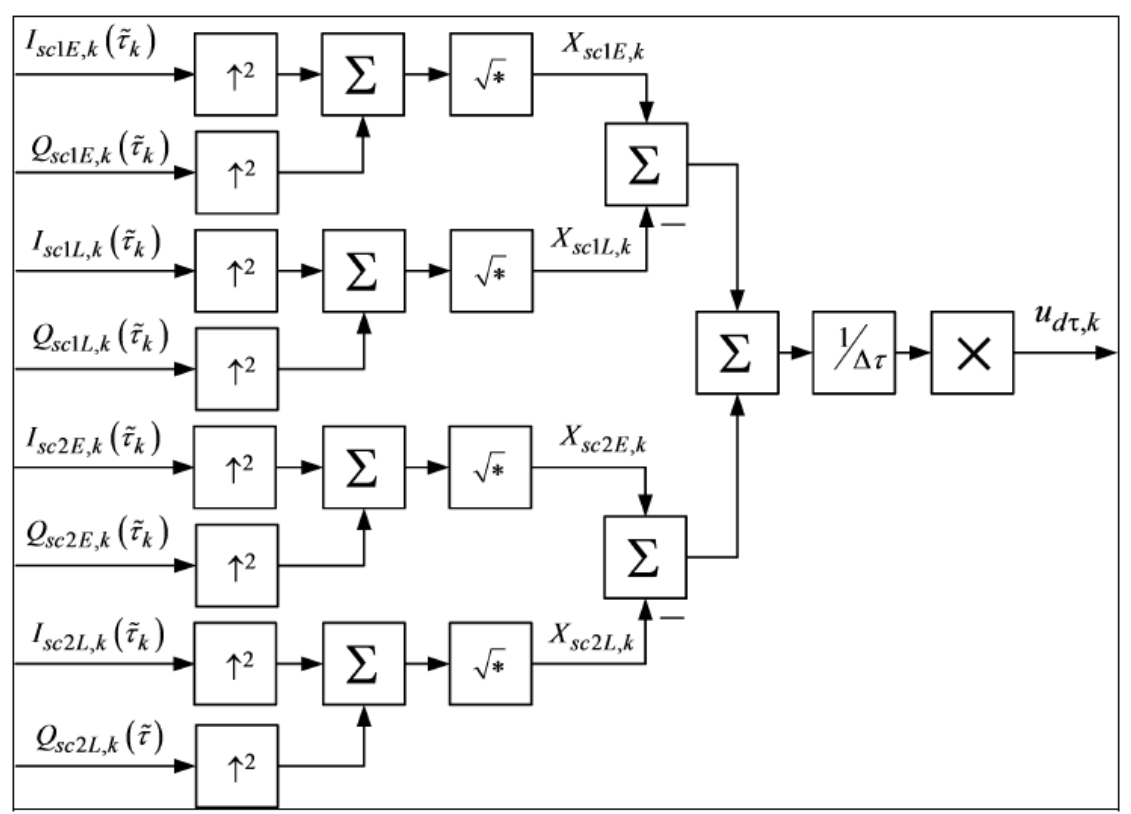

Fig. 2. Structural scheme of code delay discriminator

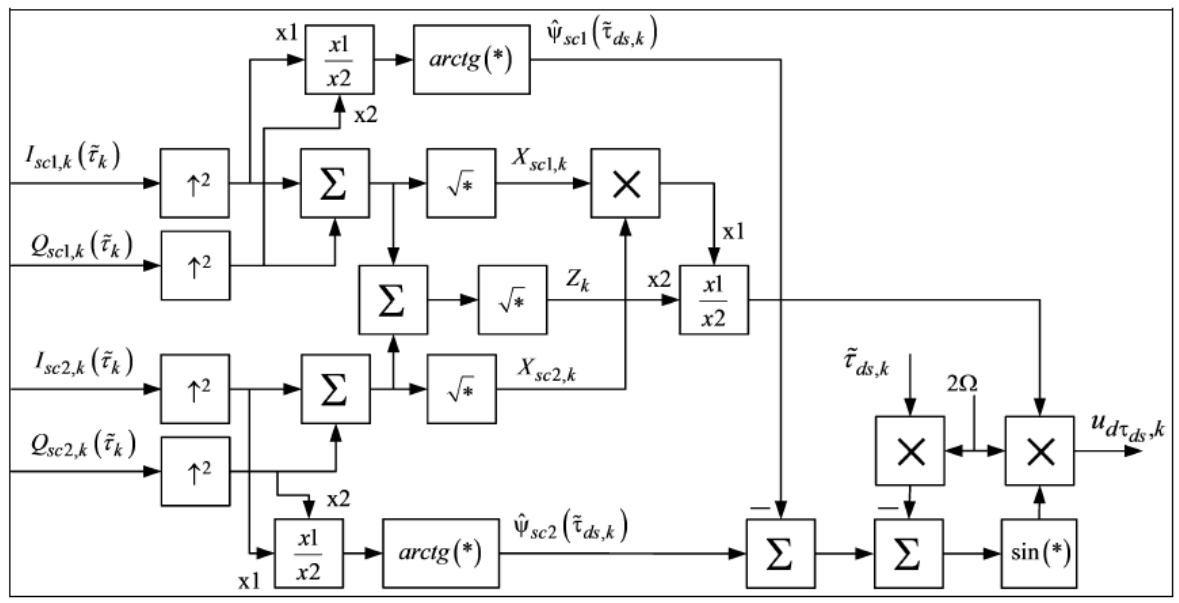

Fig. 3. Structural scheme of discriminator on dual delay variable $\tau_{d s}$

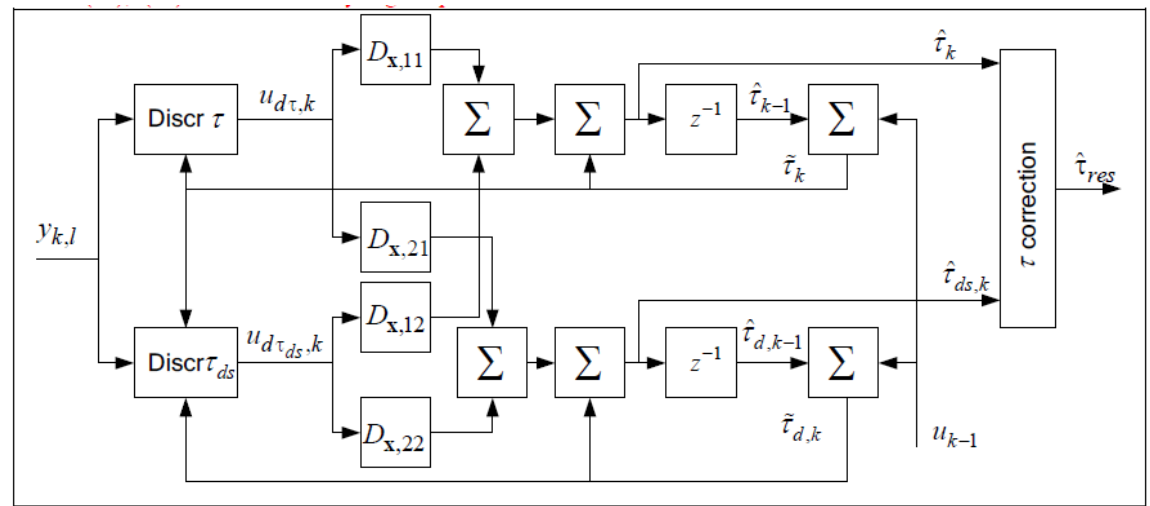

Fig. 4. Structural scheme of signal delay tracking system 
Taking into account (42) final delay estimation $\hat{\tau}_{\text {res }}$ is defined as:

$$
\hat{\tau}_{r e s}=\tau_{d s 0}(\hat{\tau})+\frac{\hat{m} T_{\Omega}}{2}
$$

The equation of optimal filtration (6) includes the derivative $\frac{\partial \tilde{F}_{k}\left(\tilde{x}_{k}\right)}{\partial x}$ for which following expression can be written:

$$
\frac{\partial \tilde{F}_{k}\left(\tilde{x}_{k}\right)}{\partial x}=\left|\frac{\partial \tilde{F}_{k}\left(\tilde{x}_{k}\right)}{\partial \tau} \frac{\partial \tilde{F}_{k}\left(\tilde{x}_{k}\right)}{\partial \tau_{d s}}\right|^{T}=\left|u_{d \tau, k+1} \quad u_{d \tau_{d s}, k+1}\right|^{T}
$$

Consequently, for tracking system the final equation can be written down in the form:

$$
\tilde{x}_{k}=\tilde{x}_{k}+D_{x, k}=\left|u_{d \tau, k+1} u_{d \tau_{d s}, k+1}\right|^{T}
$$

Thus, the synthesized processing algorithm of BOC modulated signals in incoherent operating mode of navigation receiver with processing at subcarrier frequencies is a two-loop tracking system that includes two discriminators (on an envelope delay (39) and on additional variable (40)), complex smoother filter described (44), and the algorithm of delay ambiguity resolution (42), (43) that coursed by signal periodical modulation function. For discriminator formation there are used correlators with processing at subcarrier frequencies (18), (26) and calculated on its bases match filter envelopes (20), (28).

A scheme that implements tracking on the code delay $\tau_{d}$ and on the dual delay variable $\tau_{d s}$ is shown in Fig. 4, where $z^{-1}$ designates a delay per $T$, ' $\tau$ correction' block performs the formation of final delay estimation $\hat{\tau}_{\text {res }}$ in accordance with algorithm (43).

As shown in Fig. 2 the tracking system has two discriminators and a single smoothing filter which forms estimations of the code delay $\tau$ and dual delay variable $\tau_{d s}$. This is a distinctive characteristic of synthesized tracking system with respect to known similar systems.

\section{Analysis of Discriminatory Characteristics}

Statistical characteristics of discriminators influence common property of tracking systems. Therefore let us analyze discriminatory characteristics of two synthesized discriminators.

Let us calculate mean value of in-phase component in $(18)$ :

$$
\begin{aligned}
& M\left[I_{s c 1, k}\right]=\bar{I}_{s c 1, k}=\frac{A}{2 \sigma_{n}^{2}} \sum_{l=1}^{N} A h_{c}\left(t_{k, l}-\tau_{k}\right) h_{D I}\left(t_{k, l}-\tau_{k}\right) \\
& h_{d s}\left(t_{k, l}-\tau_{k}\right) \cos \left(\omega_{0} t_{k, l}+\varphi_{k, 0}+\omega_{D, k}(l-1) T_{d}\right) \\
& \times h_{c}\left(t_{k, l}-\tau\right)=\frac{-q_{c / n 0} T b_{D I}}{\pi} \rho\left(\varepsilon_{\tau, k}\right) \sin \left(\Omega \tau_{d s, k}-\varepsilon_{\varphi 1, k}\right)
\end{aligned}
$$

where, $b_{D I}$-value of digital information symbol; $\varepsilon_{\varphi 1, k}=$ $\varphi_{k, 0}-\varphi_{r e f 1, k},=\frac{A^{2}}{2 N_{0}}=\frac{P_{c}}{N_{0}}[H z]$-signal-to-noise ratio:

$$
\rho\left(\varepsilon_{\tau}\right)=\frac{1}{T} \int_{0}^{T} h_{c}\left(t-\tau_{k}\right) h_{p}\left(t-\tilde{\tau}_{k}\right) d t
$$

where, $\varepsilon_{\tau}=\tau_{k}-\tilde{\tau}_{k}$.

Other correlation sums in (18), (26) are calculated similarly, which results in the following correlations:

$$
\begin{aligned}
& \bar{Q}_{s c 1, k}=\frac{q_{c / n_{0}} T b_{D I}}{\pi} \rho\left(\varepsilon_{\tau}\right) \cos \left(\Omega \tau_{k}+\varepsilon_{\varphi 1, k}\right), \\
& \bar{I}_{s c 2, k}=\frac{q_{c / n_{0}} T b_{D I}}{\pi} \rho\left(\varepsilon_{\tau}\right) \sin \left(\Omega \tau_{k}+\varepsilon_{\varphi 2, k}\right), \\
& \bar{Q}_{s c 1, k}=\frac{q_{c / n_{0}} T b_{D I}}{\pi} \rho\left(\varepsilon_{\tau}\right) \cos \left(\Omega \tau_{k}+\varepsilon_{\varphi 2, k}\right)
\end{aligned}
$$

It should be noted that in the statistical equivalents (44), (45) the true value of $\tau_{k}$ delay enters under the signs of trigonometric functions.

Calculate the average value of $X_{s c 1, k}$. In case of large signal-to-noise ratio it is possible to write:

$$
\begin{aligned}
& \bar{X}_{s c 1, k}\left(\tau_{k}\right)=\sqrt{\bar{I}_{s c 1, k}^{2}\left(\tau_{k}\right)+\bar{Q}_{s c 1, k}^{2}\left(\tau_{k}\right)}=\frac{q_{c / n_{0}} T}{\pi}\left|\rho\left(\varepsilon_{\tau}\right)\right| \\
& \bar{X}_{s c 2, k}\left(\tau_{k}\right)=\sqrt{\bar{I}_{s c 2, k}^{2}\left(\tau_{k}\right)+\bar{Q}_{s c 2, k}^{2}\left(\tau_{k}\right)}=\frac{q_{c / n_{0}} T}{\pi}\left|\rho\left(\varepsilon_{\tau}\right)\right|
\end{aligned}
$$

It follows from (46), (47) that average values of envelopes do not depend on the mismatches between phases $\varepsilon_{\varphi 1, k}$ and $\varepsilon_{\varphi 2, k}$ between input and reference HF oscillations.

With regard to (46), (47) the discriminatory characteristic of delay discriminator (39) is written in the form:

$U_{d \tau}=\frac{2 q_{c / n_{0}} T}{\pi \tau_{c}}\left(\left|\rho\left(\varepsilon_{\tau}-\Delta \tau / 2\right)\right|-\left|\rho\left(\varepsilon_{\tau}+\Delta \tau / 2\right)\right|\right)$ 
Suppose $\Delta \tau=\tau_{c}$. Then for the slope of the discriminatory characteristic the following formula can be written down:

$$
S_{d \tau}=\frac{4 q_{c / n_{0}} T}{\pi \tau_{c}^{2}}
$$

Let us calculate mathematical expectations:

$$
E\left[\hat{\psi}_{s c 1}\right]=-\Omega \tau_{d s}, E\left[\hat{\psi}_{s c 2}\right]=\Omega \tau_{d s}
$$

Consequently, the discriminatory characteristic of the dual delay variable $\tau_{d s}(40)$ is written in the form:

$$
U_{d \tau_{d s}}=\frac{\Omega q_{c / n_{0}} T}{\pi} \sin \left(2 \Omega\left(\tau_{d s}-\tilde{\tau}_{d s}\right)\right)=\frac{2 q_{c / n_{0}} T}{\tau_{c}} \sin \left(2 \Omega\left(\tau_{d s}-\tilde{\tau}_{d s}\right)\right)
$$

The discriminator curve slope for the dual delay variable discriminator is described by the formula:

$S_{d \tau_{d s}}=\frac{4 \Omega q_{c / n_{0}} T}{\tau_{c}}=\frac{8 \pi q_{c / n_{0}} T}{\tau_{c}^{2}}$

It follows from comparison of (48) and (49) that discriminator curve slope for the dual delay variable discriminator is by $2 \pi^{2}$ times more than that of delay discriminator. Given that the potential accuracy of the delay estimation is inversely proportional to the discriminator curve slope, it can be concluded that the use of the dual delay variable discriminator provides for the increased accuracy of delay tracking.

\section{Simulation Results}

Simulations have been performed using MATLAB modeling environment. The following conditions are used for modeling:

$$
\bar{q}_{c / n_{0}}=\log \left(q_{c / n_{0}}\right)=40 d B H z ; T=8 \mathrm{~ms}
$$

At the first stage the delay modeled as stochastic process is described by (3), Doppler frequency $\omega_{D, k}$ is known and the filtering systems are supposed with constant parameters, i.e., it is described by unsteady-state equations (44), (7). Let us suppose $D_{\xi}=0,2 T \mathrm{~m}^{2}$. Figures 5 and 6 show realizations of delay tracking errors for two systems: one of them is the known tracking system with processing at the carrier frequency; another one is a synthesized tracking system with processing at the subcarrier frequency. Dash lines in the figures indicate band edges of confidence interval $\pm 3 \sigma_{\tau}$ where $\sigma_{\tau}$-root mean square of delay tracking error calculated using Riccati Equation 7.

Root mean square values of the delay tracking error calculated by realizations of Fig. 5 and 6 are equal to $\sigma_{\tau, s c}=0.4$ and $\sigma_{\tau, s c}=0.54 \mathrm{~m}$, respectively.

Root mean square values of the delay tracking error calculated by realizations of Fig. 5 and 6 are equal to $\sigma_{\tau, s c}=0.4$ and $\sigma_{\tau, s c}=0.54 \mathrm{~m}$, respectively.

Figures 7 and 8 show similar tracking errors at stronger dynamics of delay change $D_{\xi}=4 T \mathrm{~m}^{2}$.

Root mean square values of the delay tracking error calculated by realizations of Fig. 7 and 8 are equal to $\sigma_{\tau, s c}=0.78$ and $\sigma_{\tau, s c}=1.21 \mathrm{~m}$, respectively.

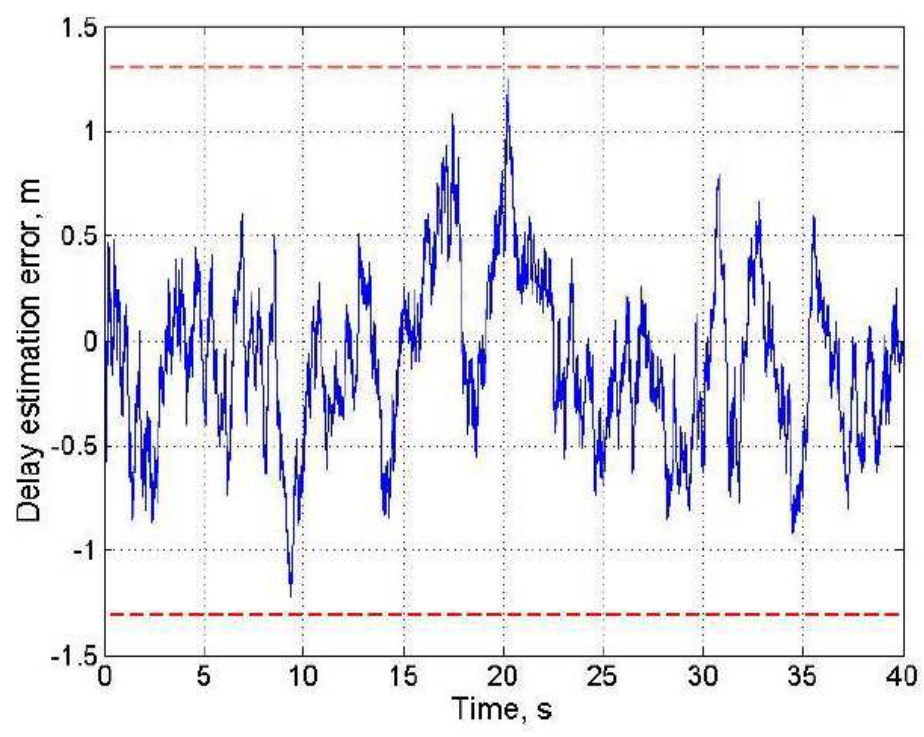

Fig. 5. The delay tracking error in the tracking system with processing at subcarrier frequencies when $D_{\xi}=0,2 T \mathrm{~m}^{2}$ 


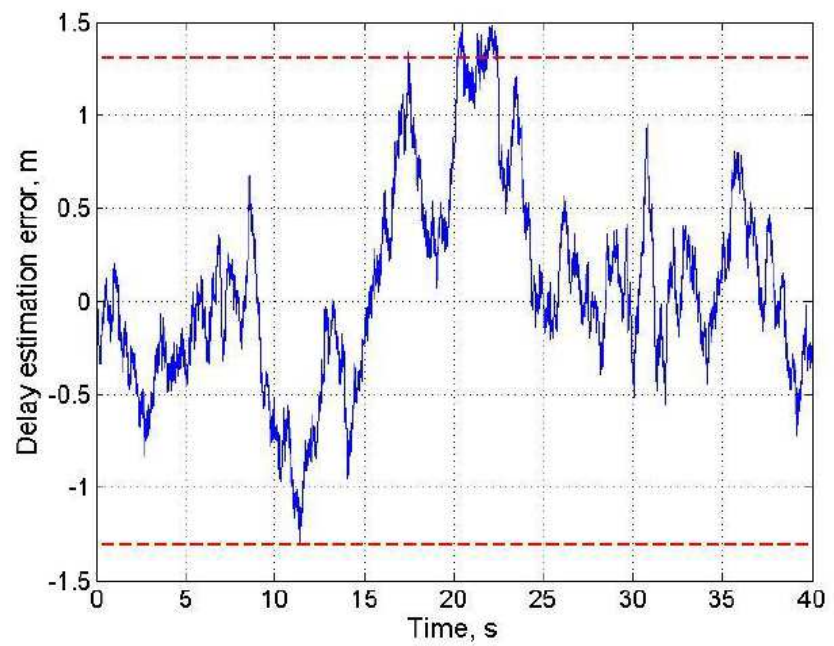

Fig. 6. The delay tracking error in the tracking system with processing at the carrier frequency when $D_{\xi}=0,2 T \mathrm{~m}^{2}$

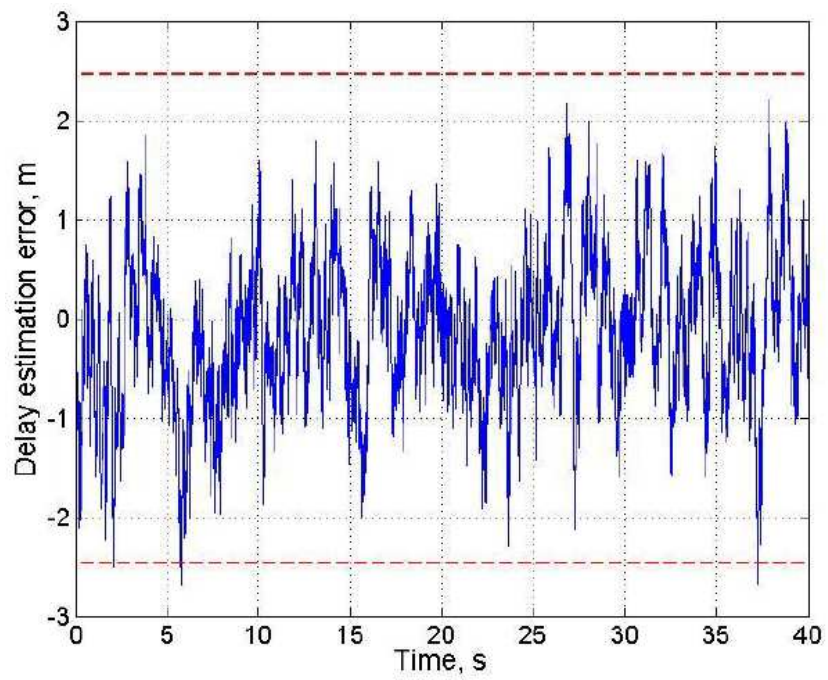

Fig. 7. The delay tracking error in the tracking system with processing at subcarrier frequencies when $D_{\xi}=4 T \mathrm{~m}^{2}$

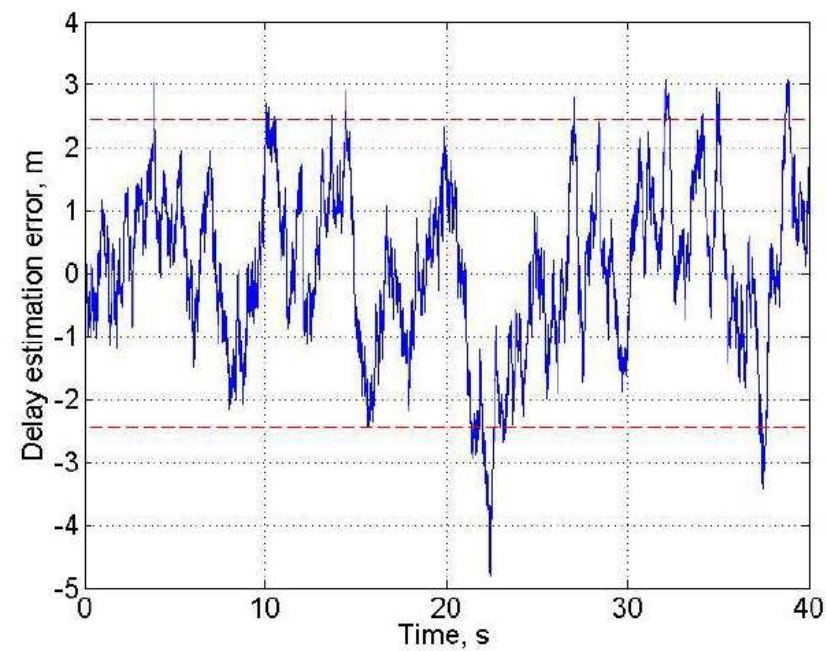

Fig. 8. The delay tracking error in the tracking system with processing at the carrier frequency when $D_{\xi}=4 T \mathrm{~m}^{2}$ 
It follows from simulation results that synthesized racking system with processing at subcarrier frequencies provides delay tracking rms by 1.4-1.6 times less then known tracking system with processing at the carrier frequency when receiving $\operatorname{BOC}(1,1)$ modulated navigation signal.

\section{Conclusion}

Subcarrier processing technique for incoherent operating mode of navigation receiver is derived using optimal filtering theory and dual delay estimation method. Equations for tracking system have been obtained that include two discriminators and common integrated filter. Discriminatory characteristics are calculated. It is shown that these characteristics have stable balance point that provides stable tracking. The slope of discriminatory characteristic for the dual delay variable discriminator is by $2 \pi^{2}$ times more than that of the delay discriminator. Structural schemes of the synthesized discriminators are given. It is indicated that in the synthesized tracking system with processing at subcarrier frequencies the root mean square value of the signal delay error is about 0.4$0.8 \mathrm{~m}$ for different delay dynamics and it is by 1.4-1.6 times less than the similar error in the tracking system with processing at carrier frequency.

\section{Acknowledgment}

The authors would like to thank the anonymous reviewers and the editor for their insightful comments and suggestions.

\section{Ethics}

This article is original and contains unpublished material. The authors confirm that there are no ethical issues involved.

\section{References}

BeiDou NSS, 2013. BeiDou navigation satellite system signal in space interface control document open service signal (Version 2.0).

Chen, S., K.H. Thiol and A. Kleusberg, 2006. Implementation and analysis of acquisition and tracking algorithms for BOC signals. Proceedings of the 19th International Technical Meeting of the Satellite Division of the Institute of Navigation, Sept. 26-29, Fort Worth, TX, pp: 1821-1829.

De Castro, D., J. Diez, A. Fernández and J.M. Sleewaegen, 2006. A new unambiguous low-complexity BOC tracking technique. Proceedings of the 19th International Technical Meeting of the Satellite Division of the Institute of Navigation, Sept. 26-29, Fort Worth, TX, pp: 1830-1835.
European GNSS, 2010. Open service signal in space interface control document. European GNSS.

Fante, R.L., 2003. Unambiguous tracker for GPS binaryoffset-carrier signals. Proceedings of the 59th Annual Meeting of the Institute of Navigation and CIGTF 22nd Guidance Test Symposium, Jun. 23-25, Albuquerque, NM, pp: 141-145.

Fine, P. and W. Wilson, 1999. Unambiguous tracker for GPS binary-offset carrier signals. Proceedings of the National Technical Meeting of the Institute of Navigation, (MIN' 99), San Diego, CA, pp: 671-676.

Gyu-In, J., I. Sung-Hyuck and I. Byimg-Hyun, 2007. Optimal code and carrier tracking loop design of Galileo BOC(1,1). Proceedings of the 20th International Technical Meeting of the Satellite Division of The Institute of Navigation, Sept. 25-28, Fort Worth, TX, pp: 759-768.

Heiries, V., D. Roviras, L. Ries and V. Calmettes, 2004. Analysis of non ambiguous BOC signal acquisition performance. Proceedings of the 17th International Technical Meeting of the Satellite Division of the Institute of Navigation, Sept. 21-24, Fort Worth, TX, pp: 2611-2622.

Hodgart, M.S., P.D. Blunt and M. Unwin, 2007. The optimal dual estimate solution for robust tracking of Binary Offset Carrier (BOC) modulation. Proceedings of the 20th International Technical Meeting of the Satellite Division of The Institute of Navigation, Sept. 25-28, Fort Worth, TX, pp: 1017-1027.

Hodgart, M.S., R.M. Weiler and M. Unwin, 2008. A triple estimating receiver of Multiplexed Binary Offset Carrier (MBOC) modulated signals. Proceedings of the 21st International Technical Meeting of the Satellite Division of The Institute of Navigation, Sept. 16-19, Savannah, GA, pp: 877-886.

IS-GPS-200F, 2011. Interface Specification GPS Space Segment. User Segment.

IS-GPS-800B, 2011. Interlace specification GPS Space Segment. User Segment L1C Interfaces.

Jovancevic, A., S. Ganguly, B. Sirpatil, M. Kirchner and S. Zigic, 2007. Real-time software based block processing m-code receiver. Proceedings of the 20th International Technical Meeting of the Satellite Division of The Institute of Navigation, Sept. 25-28, Fort Worth, TX, pp: 142-151.

Jovanovic, A., C. Mongrédien, Y. Tawk, C. Botteron and P.A. Farine, 2012. Two-step Galileo E1 CBOC tracking algorithm: When reliability and robustness are keys! Int. J. Navigat. Observat.

DOI: 10.1155/2012/135401

Martin, N., V. Leblond, G. Guillotel and V. Heiries, 2003. BOC $(x, y)$ signal acquisition techniques and performances. Proceedings of the 16th International Technical Meeting of the Satellite Division of the Institute of Navigation, Sept. 9-12, Portland, OR, pp: 188-198. 
Musso, M., A.F. Cattoni and C.S. Regazzoni, 2006. A new fine tracking algorithm for binary offset carrier modulated signals. Proceedings of the 19th International Technical Meeting of the Satellite Division of the Institute of Navigation, Sept. 26-29, Fort Worth, TX, pp: 834-840.

Perov, A.I. and V.N. Kharisov, 2010. GLONASS: Principles of Constructing and Functioning. 4th Edn., Radiotekhnika, Moscow.

Perov, A.I., 2012. Methods and algorithms of optimal signal reception in the users' equipment of satellite radio navigation systems. Radiotehnika, Moscow.

Perov, A.I., 2015. Delay tracking algorithm for the pilot component of GLONASS perspective signal with code division multiple access. Modern Applied Sci., 9: 144-161. DOI: 10.5539/mas.v9n5p144
Radiotekhnika, 2012. Fundamentals of constructing radio-navigation satellite systems. Radiotekhnika, Moscow.

Tikhionov, V.I. and V.N. Kharisov, 1984. Integral synchronization in radio technical systems. Radio Eng., 39: 3-10.

Tikhionov, V.I. and V.N. Kharisov, 2004. Statistical analysis and synthesis of wireless devices and systems. Moscow.

Vinogradov, A.A., A.E. Perkov and A.O. Rodchenko, 2009. Estimation algorithms of GNSS advanced signal delay with BOC-modulation based on supplementary variable technique. Radio Eng., 7: 99-104. 